\title{
ANÁLISIS COMPARATIVO PARA LA ECONOMÍA MAGALLÁNICA DESDE LA PERSPECTIVA DE LA SENSIBILIDAD DE COEFICIENTES TÉCNICOS.
}

\author{
SERGIO SOZA-AMIGO","
}

\section{RESUMEN}

Este trabajo compara diferentes medidas basadas en sensibilidades estructurales de la Región de Magallanes con las Regiones de Tarapacá, del Libertador Bernardo O'Higgins Riquelme, del Bío-Bío, y de Los Lagos. El objetivo es mostrar cuales son los cambios en las actividades que afectan significativamente la producción de las regiones estudiadas, de manera de poder establecer las similitudes en las respuestas a cambios externos de las regiones estudiadas.

Utilizando la técnica de límites tolerables, y las tablas de insumo- producto, desarrolladas por el INE-Chile y posteriormente modificadas por MIDEPLAN, primero se presenta un análisis de sensibilidad de las etapas productivas empleando la propuesta de Schintke y Stäglin (1988) para identificar la importancia de cada actividad o rama. Luego, se obtienen los campos de influencia a través del uso de la teoría de Grafos (Aroche, 1996; 2002 y 2005). Finalmente, se concluye que, al existir una mayor interacción de las actividades, aumentan las alternativas que permiten acceder a un desarrollo económico exitoso, ya que el grado de interacción espacial al interior de una región, se asocia a un abanico de posibilidades que permiten un crecimiento de la economía.

PALABRAS CLAVES: Análisis Estructural, Análisis de Sensibilidad, Coeficientes Importantes, Campos de Influencia.

\section{Clasificación JEL}

C67; D57; R15; R34 y Z00.

* Universidad de Magallanes, Facultad de Ciencias Económicas y Jurídicas, Av. Bulnes 01855, Punta Arenas, Chile, e-mail: sergio.soza@umag.cl.

* Se reconoce y agradece el apoyo del FONDECYT, proyecto número 11070168: "Análisis Estructural de la Economía Magallánica Desde la Perspectiva del Enfoque Input-Output" y, del Núcleo de la Iniciativa Científica Milenio "Ciencia Regional y Políticas Públicas". 


\title{
COMPARATIVE ANALYSIS FOR THE ECONOMY MAGALLANES FROM THE PERSPECTIVE OF THE SENSIBILITY OF TECHNICAL COEFFICIENTS
}

\begin{abstract}
The aim of this paper is to show how changes in sectoral activities affect the output of some of the Chilean regions. The Magellan region is compared to other three regions: Libertador Bernando O’Higgins, Bío Bío and Los Lagos to assess similarities and differences among them.

Using the field of influence techniques, and the Chilean regional Input-Output tables (INE and MIDEPLAN), according to Schintke y Stäglin (1988) to identify the key sectors and treating the results with tools from graph theory (Aroche, 1996; 2002 y 2005). Finally, it can be it concluded that, when a major interaction of the activities exists, they increase the alternatives that they allow to accede to an economic successful development, since the degree of spatial interaction to the interior of a region associates with a range of possibilities that allow a growth of the economy.
\end{abstract}

KEY WORDS: Structural Analysis, Linkage, Sensible Analysis, Important Coefficients, Influence Fields.

\section{JEL Classification}

C67; D57; R15; R34 and Z00.

\section{INTRODUCCIÓN}

La motivación de este trabajo, nace del interés por observar las similitudes que manifiestan determinadas estructuras económicas. En concreto, este trabajo se focaliza en el análisis de las singularidades que presentó la región de Magallanes y la Antártica Chilena ${ }^{1}$ durante 1996, frente a las particularidades que, en igual fecha, mostraban otras regiones que se consideran importantes para dicho ejercicio (Tarapacá, la del Libertador Bernardo O'Higgins Riquelme -en adelante, "O'Higgins"-, del Bío-Bío, y de Los Lagos). Para tal efecto, se recurre al marco input-output que entronca con el análisis estructural. Utilizando la mencionada metodología, se estudian las similitudes que presentan las regiones materia de estudio, empleando como elementos comunes y diferenciadores, las sensibilidades de las distintas etapas productivas que presenta cada región.

Desde la perspectiva anterior, se detectan las semejanzas y diferencias estructurales que presentan las mencionadas regiones. El identificar, por un lado, qué etapas productivas; $y$, por otro, qué ramas son las más sensibles en una economía debido a

1 En adelante región de Magallanes, Magallánica o, simplemente Magallanes. las posibles modificaciones que en ellas se puedan realizar, es un tema recurrente en un entorno de análisis input-output. Lo que al entender del suscrito, sería causado por dos razones: la primera, tendría que ver con la identificación de cuál o cuáles partes del proceso o etapa productiva, permiten alcanzar un mayor efecto sobre el resto del sistema al que pertenecen, pues una pequeña alteración en uno o más de ellos puede significar el causar efectos importantes en el desarrollo de un sector; y, en segundo lugar, debido a la importancia y, en consecuencia, a las repercusiones que puede causar el modificar una rama altamente sensible sobre el entorno económico, ya que éstas, bajo la premisa anterior, son vistas como un cúmulo de etapas productivas; por lo cual, se amplifican sus repercusiones en términos de impacto sobre el resto 2 .

Se ha optado por el análisis input-output, dado a que el mismo proporciona una amplia y profunda visión de la estructura económica que se aborda. En una tabla input-output [TIO o matriz insumo- producto (MIP)], se muestra por un lado, la cuantificación de las relaciones de compra y venta que se dan entre las distintas ramas, y por otro, la de

2 Aún cuando, la definición de "rama" es distinta de "sector", en este documento ellas son empleadas como sinónimos. 
sus inputs primarios (sueldos y salarios, beneficios, amortizaciones, excedente bruto de explotación, impuestos a la producción), así como también, su demanda final (consumo privado, consumo público, formación de capital y exportaciones). Análogamente, también se encuentra información relativa a la interrelación que presenta la economía con otros países o regiones, por medio de las importaciones. En este sentido, una de las singularidades que presenta la técnica señalada, es el ser una herramienta muy útil, en especial, cuando se trata de estudios basados en comparaciones económicas.

Otra de las causas que explicarían el empleo de las TIO, se debe a la evaluación muy aproximada y detallada de los posibles cambios que se pueden dar en una economía, por ejemplo, cuando se está frente a un shock económico, ya sea de demanda $\mathrm{u}$ oferta. La metodología en cuestión, no sólo facilita el análisis, sino que además, permita observar con más precisión las consecuencias de dicho impacto en las funciones de producción y distribución de las distintas ramas, ecuaciones que generalmente se omiten en otros modelos.

Desde otra perspectiva, esta metodología permite simular entre otras cosas, el efecto que tiene, por ejemplo, el aumento de una unidad monetaria en la demanda final (u oferta) de una economía. Bajo esta condición, se puede simular cuál será la consecuencia de dicho incremento; esto es, se obtienen respuestas, que no sólo señalan la medida en que aumenta la producción en su conjunto, sino que muestra en qué ramas y, en concreto, qué etapas productivas se ven más afectadas con dicho incremento. Luego, y bajo esta premisa, un planificador regional podría evaluar, todas las cosas consideradas, cuál será el impacto de una política que afecte directamente a las exportaciones, o bien, podría analizar qué ocurriría en términos aproximados, si se establece una alteración en los salarios, o bien la aplicación o eliminación de un determinado impuesto. Como se puede apreciar, estas son algunas de las alternativas que facilitan el tener que elegir qué ramas o actividades económicas potenciar. En este sentido, estas son algunas de las simulaciones que, sin duda, ayudan a comprender de mejor manera las consecuencias de un cambio, sobre todo, si se tiene en cuenta que el actual entorno económico es cada vez más inestable. Luego, este tipo de técnicas ayudan a evaluar dichas alteraciones en forma muy aproximada y rápida.
Como se puede observar, un análisis estructural ayuda a comprender de mejor manera las consecuencias que tiene la implementación de políticas económicas, dado que induce y estimula el uso de la simulación de medidas económicas bajo un contexto caeteris paribus, a lo que se puede agregar, el facilitar un parangón más detallado de las estructuras que se desean analizar, permitiendo de esta forma -aunque no garantizando-, el aumento de las posibilidades de lograr un cierto éxito económico, o bien, evitar un fracaso, basados en la aplicación de medidas y experiencias tomadas a partir de la TIO de otro lugar. Por ejemplo, dada una medida que permitió alcanzar un cierto éxito económico, esto es, una decisión o política económica que se aplicó a una determinada actividad, que podemos designar como la i-ésima-, en la región $r$-ésima, se observa las repercusiones que tiene tal implementación, en la región n-ésima, que es la que se analiza. En otras palabras, se simula la decisión, que sería tomada en la región r-ésima, para la actividad i-ésima, en el sector i-ésimo de la n-ésima localidad, para posteriormente observar sus principales repercusiones.

La razón del porqué se compara Magallanes con las citadas regiones, obedece, para el caso de la región de Tarapacá, a la condición de zona extrema que ambas comparten. Adicionalmente, se hace atractivo comparar Magallanes con la región del Libertador Bernardo O’Higgins y la de Los Lagos, dado el alto crecimiento económico que estas han manifestado en los últimos años, y además por que esta última tiene un elevado desarrollo del sector turismo, y por último, cotejarla con la región del Bío-Bío, en base a la diversidad económica que esta última manifiesta (Banco Central, 2008; 2007 y 2006 y, MIDEPLAN, 2005) ${ }^{3}$.

$\mathrm{Si}$ se admite que los resultados emanados de un análisis, cuyo sustento es la metodología input- output, pueden servir como plataforma para la implementación de futuras políticas económicas, se cree que, si tal estudio, además presenta como característica el apuntar a la identificación de las similitudes estructurales económicas que existen entre distintas zonas geográficas (dentro de las cuales existen dos que son extremas), este nutrirá más, en términos de información, al planificador regional. Lo planteado, obedece

3 Se excluye del análisis a la región de Aysén (XI), que es colindante con Magallanes, ello obedece a la pobre actividad económica que la primera manifiesta frente a la última. 
a que, tras realizar tal contraste, se obtendrán, por un lado, respuestas tales, que faciliten la comprensión de las consecuencias que causa el modificar las etapas productivas que se representan en una TIO en particular. Es decir, el ejercicio realizado, suministra información que lleva a una mejor comprensión de los resultados que se obtienen luego de modificar una etapa productiva, $y$, en segundo lugar, se observan las semejanzas y divergencias que presentan las estructuras que se comparan, luego de aplicar las mismas alteraciones para cada una de las MIP, que forman el conjunto de regiones que se comparan.

En referencia a la información estadística que se empleará, ella se deriva de tablas insumoproducto regionales publicadas por MIDEPLAN en 2005. En lo relativo a las matrices que se usarán como medio de comparación, estas son las de 4 regiones anteriormente mencionadas. Finalmente, se trabajará considerando a 40 ramas de actividad, cantidad que se estima adecuada a estos efectos ${ }^{4}$ (Soza, 2007, pp. 199), para comprender de mejor manera las semejanzas y divergencias que presentan las distintas regiones estudiadas. En anexo $1 \mathrm{~A}$, se entrega un detalle de estos sectores.

En consideración a lo expuesto, se ha fijado como un primer objetivo el desarrollar una metodología que, por un lado, sea novedosa y práctica, y por otra parte, sea capaz de facilitar un cotejo entre las distintas estructuras económicas que se deseen analizar. En este sentido, lo que se proponga debe ser capaz de combinar, en primer lugar, la información derivada del entramado de las economías analizadas, en base a los conceptos de Límites Tolerables, Campos de Influencia y Clúster Jerárquico. En segundo lugar, se aplicará la propuesta con el fin de detectar las principales semejanzas y divergencias que existen entre las regiones que se toman para el caso en estudio. En este sentido, se espera dar respuesta a un doble desafío: por un lado, está el mostrar un análisis estructural input-output del tipo comparativo en base a la metodología que se propone, $y$, por otro, el detectar las divergencias $y$ semejanzas que se presentan en las regiones, mediante la técnica de los límites tolerables, prestando especial atención a la región de Magallanes.

4 Se considera que es adecuada, básicamente por dos razones, la primera tiene que ver con el efecto senoidal que toman los elementos de la matriz inversa de Leontief tras cada agregación que se realiza $y$, el segundo, por el nivel de detalle que se logra con esa agrupación.
Planteado lo anterior, el procedimiento, se inicia abordando una primera parte, en donde se determinan las elasticidades de cada etapa productiva mediante la técnica de los límites tolerables (sensibilidad de los distintos coeficientes técnicos). Para ello se emplea la formulación presentada por Schintke en 1984, la cual es posteriormente divulgada por Schintke y Stäglin en 1988. Tal razón obedece a que las mismas se consideran más adecuadas a estos efectos (Soza, 2007, pp. 134).

En una segunda fase, y sobre la base de las respuestas derivadas de la sensibilidad que presentó cada etapa productiva, se confeccionan los campos de influencia de las distintas ramas. De esta forma, y dependiendo de cuán sensible sean las etapas que forman la función de producción que se analiza, esto es, e.g., en base al número de etapas sensibles que presentó la i-ésima rama respecto a la n-ésima, se esbozan las relaciones de oferta y demanda, obteniéndose con ello los vínculos (campos) que estas forman con su entorno, utilizando para estos fines la metodología presentada por Aroche en 1996; 2002 y 2005.

Resumiendo, en esta oportunidad se revisan las similitudes estructurales de cinco regiones, en base a la sensibilidad que presentan sus distintos coeficientes técnicos (elasticidades), y se observa cuál es el campo de influencia que forma cada actividad (interrelación de las distintas etapas productivas con el medio), para así transformar estos enfoques en herramientas y métodos que ayuden en la toma de decisiones. Desde tal perspectiva, abordar la temática propuesta, facilitará la interpretación económica de las regiones materia de estudio.

\section{Sensibilidad y Campos de Influencia}

El objetivo de este apartado es presentar la génesis y operatoria que existe tras la sensibilidad que muestran las distintas etapas productivas. En este sentido, y basados en la técnica de los límites tolerables, lo que continúa se aborda desde dos situaciones hipotéticas: en la primera, se indaga en el cómo se ve afectada la economía cuando se altera sólo una etapa de la i-ésima función de producción, esto es, cuando es únicamente el coeficiente $\mathbf{a}_{\mathbf{i j}}$ perteneciente a la i-ésima rama el alterado; y, en segundo lugar, se analiza qué ocurre cuando existe un conjunto de cambios, para lo cual se modifica cada una de las etapas que forman las funciones de producción por separado. Una vez 
realizado ello, se observa qué relaciones forma cada coeficiente, cuando éstos son modificados de forma independiente, lográndose de esta forma visualizar los campos de influencia que cada una forma, en base al número de etapas sensibles que presenta la rama.

La idea de alterar una parte de un proceso productivo, surge indirectamente de los trabajos desarrollados, a principios de los años 50, por Waugh y Sherman y Morrison. Sin embargo, fue Evans en 1954, quien llevó la idea a la práctica económica, y es Sebald en 1974, quien introduce a este esquema el concepto de "elasticidad", concepto que cuantifica la alteración de un coeficiente técnico, sobre la producción total. Posteriormente, y sobre la base de las ideas anteriores, es Schintke en 1984, y luego Schintke y Stäglin en 1988, quienes presentan una formulación para valorar en forma más precisa la elasticidad, o, si se prefiere, la sensibilidad que presentan los distintos coeficientes $\mathbf{a}_{\mathbf{i j}}$, señalando que un coeficiente será importante cuando una variación en éste, que sea menor que el 100\%, sea capaz de generar un cambio en la producción de alguna de las ramas en mayor medida que el nivel porcentual prefijado.

La formulación que presentan Schintke y Stäglin en 1988 (ecuación 1), parte de la base del efecto que se produce en una economía tras la alteración de un $1 \%$ en una de sus etapas productivas. De esta forma, la respuesta indicará cuál es el porcentaje en que debe variar el coeficiente $\mathbf{a}_{\mathbf{i j}}$, para que se produzca un cambio en la producción sectorial del 1 por ciento. En este sentido, la repuesta obtenida dará una noción de qué rama es más importante, de acuerdo al número de coeficientes más sensibles que presente, tanto en columnas como en filas ${ }^{5}$.

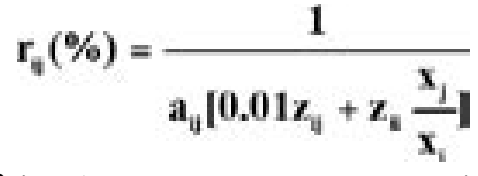

5 Bajo este esquema, una rama se considerará importante -caeteris páribus- de acuerdo al número de coeficientes $\mathbf{r}_{\mathrm{ij}}$ que presente, por ejemplo, menores que 10, tanto en columnas cómo en filas. Si una rama tiene muchos coeficientes importantes en columna, indicará lo relevante que son sus etapas productivas para la demanda de output de otros sectores. Por tanto, su importancia se asocia a las modificaciones tecnológicas del proceso de producción de las distintas ramas. Un alto número de estos coeficientes en filas, será señal de la relevancia que tienen los productos de esta actividad para el progreso de las otras; en este caso, su importancia se asociará a mejoras e innovaciones que se den en la producción (López y Pulido, 1993, pp. 176).
Posteriormente, surgieron las propuestas de Hewings et al en 1989, y la de Sonis y Hewings (1989; 1991 y 1992), investigadores que realizan un interesante aporte, cuál es la ampliación que realizan al planteamiento de Sebald (1974), llevando tal idea a pares de actividades económicas, y motivando, de esta forma, el surgimiento del concepto de "campos de influencias", cuyo objetivo central tiene por fundamento el observar qué produce la alteración de un coeficiente $\mathbf{a}_{\mathbf{i j}}\left(\forall \mathbf{a}_{\mathbf{i j}} \in \mathbf{A}\right)$, sobre la matriz inversa de Leontief. La idea intuitiva que motiva ello, se basa en la consideración de la importancia que reviste un elemento, entendido éste como una parte o etapa de un proceso productivo, para el resto del sistema económico; esto es, se detecta cuán importante es el coeficiente $\left(\mathbf{a}_{\mathrm{ij}}\right)$ para el desarrollo de otra actividad. De esta forma, si este último es importante para el primero, pasará a formar parte de lo que se denomina "campos de influencia de éste". Posteriormente, y sobre la base recientemente expuesta, Aroche en 1996; 2002 y 2005, conjugando este planteamiento con la formulación de Schnabl (1994) y la Teoría de Grafos, logra establecer, de un modo gráfico, las relaciones de dependencia de cada actividad (Soza, 2008b), idea que, como se verá más adelante, es utilizada, en parte, en este trabajo.

\section{Diseño de la Investigación}

Dada la similitud que se da entre las propuestas de West (1982, pp. 367) y la de Hewings et al (1989, pp. 72), tanto en sus planteamientos, como en sus formulaciones, y luego de comprobar que, para esta última, se pierde de vista el concepto de elasticidad desarrollada por Sebald en 1974, y por otra parte, debido a que no conjuga la variación que se plantea con la producción total, se optó por descartar las ecuaciones que propone Hewings et al, y se opta por emplear la formulación de Schintke y Stäglin (1988). Es decir, en este trabajo se toma la idea de campos de influencia desarrollada por Hewings et al en 1989, y se emplea parcialmente el planteamiento presentado por Aroche en 1996. En este sentido, lo que se hace es similar a lo desarrollado por Aroche en 1996; 2002 ó 2005, con la salvedad que, en esta ocasión, se utiliza la formulación presentada por Schintke y Stäglin en 1988, y no la de Schnabl de 1994 o, la de Holub 
y Schnabl de 1994, que son las usadas por Aroche (Aroche, 1996, pp. 237 y, 2005, pp. 34). Lo último obedece a que estas últimas expresiones son una reducción de la ecuación desarrollada por Schintke y Stäglin, y, por tanto, representa una parcelación aproximada del fenómeno estudiado.

Con el objeto de comprender las etapas que se deben cumplir para la obtención de los campos de influencia, a continuación se reproduce un ejemplo que fue presentado por Aroche en 1996 (Aroche, 1996, pp. 261), el cual es modificado con el fin de facilitar el entendimiento del proceso que se realiza. La idea que existe tras esto, es mostrar la forma en que se determinan los campos de influencia de un sector.

En el ejemplo que se presenta, se parte de la consideración que existe una matriz de coeficientes técnicos $\left(\mathbf{A}\right.$, con $\left.\mathbf{a}_{\mathbf{i j}} \in \mathbf{A}\right)$, que no ha sido alterada $y$ que posee $(4 * 4)$ elementos. Al hacer las modificaciones por separado, se obtiene un total de 16 matrices inversas de Leontief $\left(\mathbf{M}_{\mathbf{1}}\right.$ a $\left.\mathbf{M}_{\mathbf{1 6}}\right)$. Estos cambios, a su vez, quedan registrados en una matriz Booleana, la que se denomina genéricamente como matriz $\mathbf{W}$; ésta, a su vez, da origen al grafo que se reproduce más abajo, o en otras palabras, a los campos de influencia que origina cada actividad.

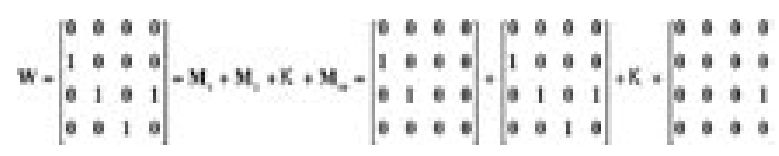

De la matriz y grafo resultante, se desprende que, si se desea que la rama número 1 aumente su producción, se requiere que, algunas de las actividades vinculada al sector número 2 -la que haya o hayan resultado ser más sensibles-, sea modificada en el valor que indique su respectivo $\mathbf{r}_{\mathrm{ij}}{ }^{6}$. De igual forma, se observa que la rama 3 , afecta a la producción de las ramas 2 y 4 . Luego, si ella es modificada, se alterará la producción de estas últimas. Además, se aprecia que existe un vínculo mutuo entre las ramas 3 y 4; esto es, para que

6 Con el fin de facilitar la interpretación de los coeficientes $\mathbf{r}_{\mathrm{ij}}$, considere el siguiente ejemplo, si un coeficiente $\mathbf{a}_{\mathbf{i j}}$ presenta un $\mathbf{r}_{\mathrm{ij}}=5$, éste se debe interpretar cómo: una variación del $5 \%$ en el coeficiente $\mathbf{a}_{\mathbf{i j}}$, con $\mathbf{a}_{\mathbf{i j}} \in \mathbf{A}$, provocaría un $1 \%$ de variación en la producción sectorial, claro está si se ha modificado todo en un 1\% (Pulido y Fontela, 1993, pp. 142).

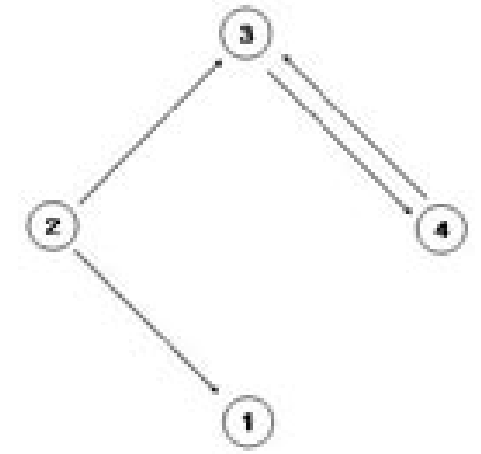

Grafo 1: Campos de influencia de cada rama, para el ejemplo tratado.

aumente la producción la rama 3 , depende de lo que ocurra con la cuatro, y lo mismo, para la 4, respecto de la 37 .

En lo que respecta al trabajo que se abordará, primero se realizará una sucesión cambios, lo que da origen a una nueva matriz por cada uno de ellos, obteniéndose de esta forma un total de 1.600 de ellas para cada zona geográfica tratada (1.600*5 en total). Luego, se confeccionan las respectivas matrices Booleanas para cada una de estas zonas. Posteriormente, y a partir de esta matriz, se confecciona los campos de influencia, en base a la técnica de grafos. Finalmente, con la información derivada del entramado de las economías, analizadas en base a los conceptos de Límites Tolerables, Campos de Influencia, se confecciona un Clúster Jerárquico, a partir del cual, se obtienen las principales semejanzas y divergencias para las regiones estudiadas.

Análisis Comparativo desde la Perspectiva de la Sensibilidad de Coeficientes Técnicos

A continuación, se hace una revisión de las actividades económicas que resultaron ser más sensibles. El criterio para su identificación ha sido el que ellas presenten elasticidades menores o

7 Por otro lado, se debe tener presente que, si bien la matriz $\mathbf{W}$, es una matriz Booleana que agrupa a un conjunto de matrices que han sido modificadas (16 en este caso), ello no significa que agrupe a todas las actividades que resultan ser altamente sensibles. Esta matriz, es simplemente una representación que dependerá de los límites que el planificador se fijó en su momento, por tal motivo, puede darse el caso que la rama 2 , tenga para otro analista más o, incluso menos de una actividad a ser modificada. 
igual a 5, esto es $\mathbf{r}_{\mathrm{ij}} \leq 5$; en otras palabras, se trata de establecer qué "etapas productivas" se deben cambiar, en forma mínima, a objeto de lograr un determinado aumento de la producción global. Para ello, se ha establecido que la variable "p", tome el valor de un 1 por ciento, por considerarse más adecuada ${ }^{8}$.

Del conjunto de regiones analizadas, llama la atención que en la región de Magallanes, la rama petróleo crudo, y combustibles y lubricantes, sea la que presentó más etapas productivas sensibles (5; ver tabla 2A en anexo); esto es, si se modifica cualquiera de esas 5 etapas, a saber, el propio autoconsumo, o el abastecimiento de otros productos alimenticios, o se establece una mejora en el trasporte, o servicios empresariales, la producción sectorial aumentaría en un $1 \%$. De estos cambios, uno de los más significativos es el del propio autoconsumo derivado de la explotación del petróleo, esto es, si dicha etapa cambia en un $3,27 \%$, la producción sectorial lo haría en un $1 \%$.

Lo anterior, es importante, ya que no siempre se observa que una actividad económica dependa en forma tan clara de las decisiones y cambios que se tomen en el propio sector para aumentar su producción, y lo es más aún, si se consideran noticias como las publicadas recientemente por la empresa GeoPark ${ }^{9}$. Ésta, en un comunicado de prensa, señala que GeoPark Holdings Limited, ..., se complace en anunciar la realización exitosa de pruebas en el nuevo pozo Manekenk 1, localizado en el Bloque Fell, Chile, cuyo volumen de producción inicial asciende a aproximadamente 1.300

8 Se ha optado por un 1\%, ya que para autores como Pulido y Fontela (1993), Aroche (1996, 2002 y 2005), Ghosh y Roy (1998), Tarancón (2003) y Robles y Sanjuán (2005) consideran que el uso del $1 \%$ es una medida habitual y, en el caso de estos últimos, en 2008 establecen que el valor estaría en el rango 0.5- 1.0\%. Otros como Siebe (1996) se decantan por un $0.5 \%$, mientras que Bullard y Sebald (1977) lo hacen por el 2\%, y Jalili (2000) llega al extremo del 10\%. En este estudio, al querer ser más conservadores se optó por el 1\%. Por otro lado, estos resultados fueron comparados con los que se obtiene de aplicar la técnica de West (1982), no encontrándose diferencias sustanciales entre ambos, pues, en general, no existe un alto número de coeficientes técnicos nulos en las TIO empleadas.

9 Noticia publicada en el World Wide Web por la Empresa GeoPark, el 1 de diciembre de 2008. Accesado el 15 de diciembre de 2008 (http://www.geo-park.com/ir/prensa. asp). barriles de petróleo equivalente diarios (bped). Por otra parte, según la Prensa Austral en su apartado Crónicas, publicado el 16 de diciembre de 2008, se informaba que, en la actualidad, la producción de Geopark alcanza los 4.700 barriles al día y que su meta es llegar a los 6.000 barriles al año; esto, más lo anterior, hacen pensar que, si aumentan esta clase de commodity, se induciría el desarrollo de la región por esta vía.

Sin embargo, se debe tener presente que, para lograr un mayor y significativo desarrollo en la región por esta vía, los nuevos yacimientos que se exploten, deben ser más importantes, pues, durante el 2007, la producción de petróleo de Magallanes estaba en torno a las $200.000 \mathrm{~m}^{3}$ (cbm). Por tanto, el incremento de la producción que se iniciará, no es muy significativo; es más, según El Mercurio (miércoles 3 de diciembre de 2008), se indica que tal yacimiento sería de tamaño medio, cito cit: Si bien, se trata del primer descubrimiento de crudo en Chile en años, la cantidad de petróleo que produce no tendría mayor relevancia. Según un experto los 1.300 barriles de petróleo diarios que pueden salir de Manekenk 1 no alcanzarían para abastecer a la planta más pequeña de ENAP en el país. Luego, y sobre la base de esta información, tal escenario, se debe analizar con más detalle, pues, al parecer, éste no debería generar mayores cambios.

Desde otra perspectiva y, sin dejar de considerar lo anterior, si adicionalmente se asume que la estructura económica de Magallanes ha experimentado pocos cambios, esto es, en consideración a la data de la TIO empleada en este trabajo, o bien estos cambios no han sido significativos, en concordancia con lo observado en terreno, para el escenario anterior, el incipiente aumento de la producción de petróleo debería repercutir con un cambio poco significativo en términos de desarrollo económico a nivel local. En este sentido, y a la luz de resultados preliminares, si bien la producción aumentará, y del mismo modo, por consiguiente, el valor agregado del sector, y también el de algunos otros que dependen del mismo (e.g., la industria ligada al gas natural y sus derivados, tales como el metanol), ello no debiera significar un alza importante en la generación de empleos y salarios, pues su efecto sobre la economía regional y nacional es muy acotado. 
En referencia al último punto, se hace ver que, las consecuencias del aumento de la producción antes mencionada, dependerán del enfoque que se emplee para su análisis, por ejemplo, si se considera a la economía como un sistema abierto o cerrado. En estas condiciones, el shock de oferta puede llegar a ser más importante en este último; de hecho, el multiplicador de la producción para este sector, cuando la economía es abierta, es de 1,64; esto quiere decir que, por cada peso en que aumenta la demanda final, el producto se incrementa en 1,64; de estos, 0,64 unidades son generados por el sistema en forma indirecta. Para cuando se considera que la economía es cerrada, este multiplicador aumenta a 2,87; esto es, si la economía lograse exportar 100 millones de pesos adicionales por este concepto por año, existiría un efecto indirecto de 64 millones; en este sentido, la producción en Magallanes aumentaría a 164 millones, por otra parte, si las rentas de esta producción quedasen en la región (economía cerrada), la producción total aumentaría a 287 millones (164 más 123), es decir, el efecto inducido por las rentas (impuestos, utilidades y salarios de quienes trabajan en la región, pero no habitan en ella) sería de 123 millones, aunque el problema está en que las rentas que se perciben por el sector no queda en la región, y por tanto, se hace necesario que exista una política que motive a que tales rentas, queden en precisamente la región donde son generados.

En base a lo anterior, se debe aclarar que, adicionalmente, hay un punto a tener en consideración: si bien es cierto hay un aumento de la producción, y seguramente tal resultado será reflejado en los respectivos valores agregados (inputs primarios), se debe recordar que, esta última variable se compone entre otros de, salarios, excedente bruto de explotación, beneficios e impuestos. Luego, si el empleo no aumenta en forma significativa, o bien, los contratados no fijan su residencia en la región -como ocurre en la actualidad-, difícilmente se podrá obtener un mayor efecto, ya que las ganancias recibidas por tales actividades, no quedarán en la región; a saber, entre otros, impuestos recibidos por el Estado, las utilidades y salarios de quienes no tiene fijada su residencia en la región. Con respecto a este último punto, se puede agregar que, además se aprecia la existencia de un alto efecto de conmutación regional, en otras palabras, lo generado en la región no queda en la región, por tanto, existe una fuga importante de los dividendos generados por el shock ${ }^{10}$.

Además de lo anterior, se observa que ramas como la 1 (productos agrícolas), 3 (ganado, productos pecuarios y carne), 4 (productos silvícolas, maderas y papel), 5 (pescados), 14 (alimentos para animales), 31 (transporte) y 35 (servicios empresariales), en el conjunto de regiones, presentan una similar cantidad de etapas productivas sensibles con respecto a Magallanes, esto es, sus estructuras dependen en gran medida de los mismos cambios para aumentar sus respectivas producciones -caeteris paribus-, como se puede apreciar. Esto, en forma adicional, da un indicio de lo parecido que puede resultar el aplicar determinadas medidas económicas, aunque claro está, ello no garantiza que sus resultados sean homogéneos en cada una de estas regiones, pero sí se acerca a las consecuencias de los mismos.

En una etapa posterior de este apartado, se revisa qué ramas albergan un mayor número de coeficientes importantes en columnas y filas. De esta forma, se diferencian los efectos de una u otra, en función de las modificaciones tecnológicas que se realicen en el proceso productivo (columnas), o los avances $e$ innovaciones que se deben presentar en otras ramas para el progreso de la i-ésima actividad (filas).

Desde la perspectiva recientemente expuesta, se observa que, en base al anexo $2 \mathrm{~A}$, la rama más importante, en general, para el conjunto de regiones (sólo lo seria en menor grado en Tarapacá), es la 3 (ganado, productos pecuarios y carne). Desde un enfoque más particular, también se aprecia que la rama que presenta más coeficientes sensibles es la 6 (petróleo crudo y combustibles y lubricantes) en la región de Magallanes, con precisamente 6 de éstos (5 en columna y 1 en fila), seguida de la región de O'Higgins con 5 de ellos en igual rama

10 Una situación similar ocurriría con la rama 19 (productos químicos, de caucho y de plástico), actividad muy vinculada a la producción de metanol, su multiplicador de producción bajo un régimen abierto es de 1,5 y, cuando se considera a la economía cerrada de 2,15 (promedio=1,38), es decir, el impacto que tendría a nivel local, si las rentas quedarán en la región sería muy alto. 
(2 y 3 respectivamente). Por otra parte, se observa que la región que más ramas importantes presenta es la de Los Lagos, en concreto las ramas 3 y 14 (ganado, productos pecuarios y carne, y alimentos para animales), poseen 5 coeficientes cada una ( 3 en columnas y 2 en filas).

Resulta importante determinar qué tan integradas estaban las actividades económicas de las distintas regiones analizadas. Ello, en consonancia con la técnica utilizada, pasa a depender del número de trazos con que cuenta su grafo, los que a su vez se limitan al marco de referencia establecido, que en este caso es el poseer actividades con una elasticidad menor o igual a $15\left(\mathbf{r}_{\mathrm{ij}} \leq 15\right)$. Esto es, una economía estará más integrada si, por un lado, cumple con esta última condición; $y$, por otro, presenta un alto número de relaciones. En tal escenario, la intuición indica que, entre más entrelazadas estén sus actividades, más afianzada y sensible a cambios en términos de desarrollo estará su economía. Desde esta perspectiva, las regiones que presentan más entrelazadas sus actividades son: las del Libertador Bernardo O'Higgins y la del Bío-Bío; posteriormente, y en forma algo menos incipiente, se encuentra la de Magallanes (anexo 2B).

También, llama la atención que la región de Magallanes presentó una estructura más entrelazada que, por ejemplo, la obtenida para la región del Bío-Bío. Lo anecdótico de este alcance, es la mayor diversidad económica y, además, el tamaño que presentó esta última región respecto a la primera, en esa fecha y que al día de hoy se mantiene. Por lo mismo, aquel escenario debe ser examinado con cautela. Bajo la actual perspectiva de análisis, tal situación es indicativa de que sus actividades no son altamente dependientes, las unas de las otras, para su desarrollo. En otras palabras, para que la economía del Bío-Bío aumente su producción, requiere que se realicen cambios muy puntuales en su estructura, no contando, por tanto, con un abanico de posibilidades mayor, como ocurre en el caso de la región de Magallanes. Desde una perspectiva similar, ello también se puede interpretar como que resulta, en términos relativos, más fácil el aumentar la producción en Magallanes, que en la región del Bío-Bío, debido a que en la zona austral, existe un mayor número de coeficientes sensibles que, al ser modificados en forma mínima, facilitan tal aumento.
Desde otro ángulo, también asombra lo poco entrelazada que se presentó la región de Tarapacá. De su grafo, se desprende lo dependiente que es su economía, ya que ésta prácticamente basó su desarrollo en una sola actividad (comercio; rama 28). Si se observan los campos que se forman en esta región, se notará que la mayoría de sus actividades están relacionas con dicho sector, dejando muy poco margen a incrementar su producción por otras vías.

En lo referente a las zonas extremas, esto es, comparando Tarapacá con Magallanes, se detecta que la economía austral era más susceptible de incrementar su producción. Aún cuando, en ambos casos sus actividades se vinculan a una misma rama (comercio), se observan diferencias. En el caso de Magallanes, se vislumbran otros tipos de cluster o vínculos de actividades, tales como, las ligadas a los servicios de hotelería y restaurantes (rama 29), o petróleo crudo y combustibles y lubricantes (sector 6). Es decir, el aumento de la productividad Magallánica, no pende de unas pocas actividades, sino que de un cúmulo de posibilidades.

\section{Similitudes Estructurales Bajo una Amplia Visión}

A continuación, se identifican qué regiones se parecen más entre ellas, tomando como criterio de semejanza los campos que forman sus actividades (tanto de demanda como de oferta), ello se realiza empleando la técnica de análisis multivariante denominada clúster jerárquico, esto último debido a la estructura de datos, y en base al propósito del acápite en cuestión. En este proceso, se han considerado a las distintas regiones como casos, y los campos que ellas forman como variables.

Como etapa previa, se ha construido una matriz que recoge las distancias entre las distintas regiones, la cual permite indagar sobre los parecidos que se dan entre ellas. Con el objeto de lograr mejores resultados, se tipificó la información. De la matriz de distancias (anexo 3A), se puede apreciar la similitud o diferencia entre las distintas regiones. La matriz es cuadrada y simétrica, y su diagonal principal es nula, porque no existe diferencia para una región consigo misma. 
La medida de distancia que se ha empleado es la distancia Euclídea al cuadrado ${ }^{11}$. De la aplicación de esta medida de divergencia, puede apreciarse que entre las regiones del Bío-Bío y la de Los Lagos es donde hay más parecido. También de allí, se desprende que existe un cierto parecido entre esta última región y la del L. B. O'Higgins. De igual forma, se constata que existen ciertas semejanzas entre los campos que se forman para las regiones de Tarapacá y Magallanes. Sobre la base de estos resultados preliminares, se puede concluir que existen ciertas semejanzas, las cuales se revisarán con mayor profundidad al construir los grupos.

Del análisis clúster jerárquico, que permite agrupar las distintas regiones de acuerdo a ciertas características (cuadro 1), en este caso, en base a los campos de influencia que posee cada una de ellas, se han obtenido tres grupos; esto es: el primero, compuesto por las regiones del Bío-Bío y de Los Lagos; un segundo, formado por la región del L. B. O'Higgins; $y$, un último, compuesto por las regiones extremas (Tarapacá y Magallanes). Resultados que pueden recogerse fácilmente de un Dendrograma -ya sea, horizontal o vertical-, puesto que se trata de una representación gráfica propia del análisis clúster, que muestra las agrupaciones que pueden constituirse (anexos 3B y $3 \mathrm{C}$ respectivamente).

Desde otra perspectiva, se observa que estos resultados son, en general, coincidentes con los presentados por Soza en 2008a. En tal investigación, se analizan las mismas regiones, pero empleando para la detección de similitudes estructurales, por un lado, el indicador de Le Masne (1988), y, por otro, las técnicas para determinar encadenamientos, presentadas por Rasmussen (1956), Dietzenbacher y van der Linden (1997) y Soza (2007; descomposición). Allí, se concluye que, en materia de similitudes y basados en el indicador de Le Masne, el mayor parangón que se obtiene para la economía

11 Cuando se determina el análisis de clúster en base a la técnica de Ward-procedimiento que tiende a combinar los conglomerados con un número reducido de observaciones-, se recomienda utilizar la distancia Euclídea al cuadrado (Hair et al, 2000, pp. 504 y 513). Sin perjuicio de lo recién comentado, se realizó el mismo análisis, pero empleando la técnica que la intuición señalaba (medidas de correlación), ya que para este caso en particular, lo que interesa, es observar los patrones que toman los valores y no sus magnitudes. De este ejercicio, se observo que no existieron diferencias entre ambos procedimiento, con lo cual se aceptan como validos ambos.
Cuadro 1: Clasificación de las regiones analizadas mediante clúster jerárquico.

\begin{tabular}{|l|l|}
\hline GRUPOS FORMADOS & Región de: \\
\hline Grupo 1 & Bío- Bío y de Los Lagos \\
\hline Grupo 2 & Del L. B. O'Higgins \\
\hline Grupo 3 & Tarapacá y Magallanes \\
\hline
\end{tabular}

Fuente: Propia en base a resultados del anexo 3B.

magallánica, se da con la región de Los Lagos, con un $81,57 \%$ de parecido. Sin embargo, las regiones del Bío- Bío y Tarapacá igualmente presentan un alto índice de correspondencia con respecto a esta región, con unos porcentajes de semejanzas igual a 79,80 y $79,39 \%$, respectivamente, muy distante de la analogía existente para Magallanes y la región del L. B. O'Higgins $(73,68 \%)^{12}$. De igual forma, y en base a la técnica elegida como instrumento de comparación (Soza, descomposición), se detectó que la mayor similitud que se dio, para el año de referencia y las regiones analizadas, se encontró para la región del Bío-Bío con la de Los Lagos, semejanza que sería coincidente con los resultados de este trabajo.

\section{CONCLUSIONES}

Lo realizado es un análisis económico estructural del tipo ex- post, para cinco regiones de Chile (Magallanes, Los Lagos, Bío- Bío, L. B. O’Higgins y Tarapacá). Para tal proceso, se utiliza el enfoque input- output. En él, se han identificado, desde distintas ópticas, cuáles fueron las actividades más importantes para estas regiones, y las similitudes que se presentan entre ellas; esto es, desde la perspectiva de la Sensibilidad de Coeficientes Técnicos (Límites Tolerables) y los Campos de Influencia que se forman en cada región. Dichos resultados permiten una mejor comprensión de las consecuencias de implementar determinadas políticas económicas,

12 En tal trabajo, adicionalmente, se indica que fueron sectores claves (ramas motivadores de demanda y oferta de insumos), bajo una visión muy restrictiva de dicho concepto, el sector 14 (alimentos para animales) en la región del L. B. O'Higgins; la 3 (ganado; productos pecuarios y carne), en la de Los Lagos, y la 6 (petróleo crudo y combustibles y lubricantes), en Magallanes, pues los resultados emanados de las técnicas empleadas (Rasmussen, Dietzenbacher y van der Linden y, Soza), resultaron indicar simultáneamente ello en tales casos, independientemente de lo que tales indicadores determinan. 
esto es, bajo el concepto de desarrollo de Albert Hirschman (1958), y considerando el supuesto caeteris páribus, si se potencian las denominadas actividades importantes, se esperaría un cierto nivel de desarrollo regional.

Del estudio de la sensibilidad de coeficientes técnicos, se detectó que la rama petróleo, combustible y lubricantes en Magallanes, presentaba la elasticidad más importante. Adicionalmente se identificaron las ramas más significativas (importantes) de acuerdo al número de coeficientes altamente sensibles, tanto en columnas como en filas. Bajo esta condición, las más vitales resultaron ser; la 6 (petróleo crudo y combustibles y lubricantes) en la región de Magallanes, con 5 coeficientes en columna y 1 en fila; dicho sector también resulto ser relevante para el desarrollo de la región de O'Higgins, pues en ella se detectaron 5 de estos coeficientes ( 2 y 3 respectivamente).

Por otra parte, se observa que la región que más ramas importantes presentó es la de Los Lagos, en concreto en las ramas 3 y 14 (ganado, productos pecuarios y carne $y$, alimentos para animales), pues en cada una de éstas, se encontraron 5 coeficientes ( 3 en columnas y 2 en filas).

Una vez modificado, por separado, cada uno de los coeficientes técnicos, lo que significo procesar 1.600 matrices de 40 ramas cada una, por cada región (1.600*5 en total), se construyó, por medio de la teoría de grafos, los campos de influencia de cada actividad, de esta etapa, y se observó cuán interconectadas están las actividades de cada región, concluyéndose que, las regiones que presentan más entrelazadas sus actividades son: las del Libertador Bernardo O'Higgins y la del Bío-Bío; posteriormente, $y$ en forma algo menos incipiente, se encuentra la de Magallanes.

Desde otra perspectiva, en base a los campos de influencia obtenidos para cada región, y empleando la técnica de clúster jerárquico, se determinó que las regiones más parecidas entre si son la del Bío- Bío con la de Los Lagos, cuyas estructuras, a su vez, se parecen a la de la región del L. B. O'Higgins. Por último, se detectó que las regiones extremas (Tarapacá y Magallanes) guardan un cierto parecido estructural, respuestas que serian coincidentes con las entregadas por Soza en 2008a.

Con respecto al procedimiento empleado, se es de la opinión que el mismo es interesante y práctico, pues las ramas que forman el entramado de las economías analizadas, son estudiadas con un planteamiento que conjuga los conceptos de Límites Tolerables con Campos de Influencia y Clúster Jerárquico, a partir de donde se obtienen las principales semejanzas y divergencias. En este sentido, lo realizado tiene la particularidad de ofrecer como ventaja posterior, la aplicación de replicar ciertas políticas, que han permitido alcanzar un determinado éxito en términos de desarrollo, en las estructuras económicas de otras regiones que presentan ciertas condiciones de similitud respecto a las que se analizan. En este sentido, se cree que las distintas propuestas que de este tipo puedan surgir, ayudan a potenciar las decisiones que se tomen en los distintos niveles locales, pues ellas contarían con un cierto respaldo exitoso. Esto último, más la utilidad de presentar una metodología que permite indagar desde otra perspectiva sobre los parecidos de las estructuras económicas, facilitarían el aplicar tales medidas a otras zonas geográficas.

\section{Campos Abiertos y de Discusión}

Debido a la matización natural que surge de los datos, y que, por tanto, afectan a las conclusiones aquí expresadas, se es de la opinión que se puede ahondar aún más en esta temática; por ejemplo, planteando un análisis estructural que abarque un cierto horizonte de tiempo. Una alternativa sería actualizando las TIOs empleadas, o bien, buscando algún medio por el cual se pueda generar información primaria regional que permita lo anterior.

En relación a la técnica escogida para la comparación (input- output, sensibilidad de los coeficientes técnicos, límites tolerables y campos de influencia), si bien es cierto puede causar controversias su uso, se cree que tal discusión puede ser originada por la falta de conocimiento del proceso en cuestión. El análisis de sensibilidad estructural, trata específicamente de dar una respuesta sistemática a la pregunta de cuál es la importancia relativa de cada uno de los elementos que componen el modelo en cuestión (oferta y demanda en su conjunto y por separado), este argumento no debe confundirse con la información que expresan los coeficientes técnicos (o su inversa), si bien es cierto tal información es relevante desde la perspectiva de la importancia relativa de las distintas ramas para comprender 
las transacciones interindustriales, ello no significa que un elemento nulo (o muy pequeño) muestre la ausencia o baja influencia que ejerce la j-ésima rama sobre la i-ésima.

Entre las bondades que se desprenden del análisis de sensibilidad, se pueden indicar las que resumen Schintke y Stäglin (1988, pp. 59) y Tarancón (2003, pp. 69): sirve para compilar, actualizar y predecir tablas input-output y facilita la comparación con otras economías. Por otro lado, se considera que la principal ventaja de este enfoque consiste en la obtención de aquellas transacciones intersectoriales que proporcionan más dinamismo a la economía, por ello, esta técnica es un buen instrumento para detectar las diferencias y similitudes estructurales que se dan entre las economías que se investiguen. En este sentido, se es de la opinión que la técnica inputoutput es la mejor para este tipo de estudios, pues es la única que relaciona funciones de producción e intercambios de oferta y demanda de cada rama con su entorno, y además, vincula los posibles efectos de los cambios de la demanda (incluyendo oferta) o inputs primarios con la economía que se analiza, sin mencionar el hecho que las tablas input- output son el origen de las cuentas nacionales de cualquier país o, región, si fuese el caso.

Prueba de lo expresado en el párrafo anterior $y$, en esa misma línea de trabajo son por nombrar sólo algunos los ya clásicos artículos de Chenery y Watanabe (1958) o más recientemente los de Sonis et al (1995) o, Dietzenbacher y van der Linden (1997) o, Aroche (1996; 2002 y 2005) o también, los de Aroca (2001) o, Robles y Sanjuán (2005a y b y 2007), o bien, los presentados para las economías europeas por Soza y Ramos en 2005 o por Soza en 2007 y 2008a y b y 2009a y b, y por último, los de Paul De Boer (enero de 2008), o los de Akiko Nakajima (septiembre de 2008) y el de Jan Oosterhaven, Dirk Stelder y Satoshi Inomata (diciembre de 2008), estos últimos todos presentados en la revista especializada de la disciplina respectiva (Economics Systems Research), todo ello dado que la técnica permite extrapolar conclusiones en base a lo ocurrido, pues su base reposa sobre funciones de producción y no, en eventuales indicadores, que no explican en detalle como dichas funciones o procesos productivos se ven afectados cuando cambia un componente de la oferta y/o demanda de la rama o actividad que se estudia, o bien, del conjunto del sistema productivo, en este sentido, la técnica además es muy utilizada para medir ciertos efectos, por ejemplo, el cambio en las tasas impositivas; o bien, para predecir y simular determinadas y variadas políticas económicas y sociales. Errado sería concluir que la técnica sólo sirve para entender la dinámica pasada y no los cambios que se puedan presentar a futuro, pues además es sabido que los cambios estructurales tardan en efectuarse, tal demora puede llegar a tomar incluso años, lo que dependerá de la variable que se emplee para motivar dicho cambio, esto es, elementos de demanda, oferta o producción, las cuales dependerán del énfasis y velocidad con que se quiera lograr la variación. En consonancia con lo plateado, sólo indicar que, en general, los países desarrollados utilizan cómo base, esta técnica para fijar sus políticas de corto y largo plazo ${ }^{13}$.

En lo referente a las implicaciones que tendrían ciertas respuestas, como por ejemplo, lo sensible que resulta ser la rama petróleo y sus derivados, las mismas se deben entender dentro del marco tratado, esto es, una economía doméstica, en donde se excluye el mercado externo pero se incorporan los efectos locales (resto de regiones), por tanto, se incorporan tácitamente los cambios de la demanda y oferta nacional y local, en este sentido, las respuestas derivadas del análisis, sirven para apoyar variadas Políticas Económicas, las que en algunas ocasiones erróneamente se basan en, por ejemplo, sólo en el PIB -baste recordar que dicho indicador es un componente de las tablas input-output-, el cual si se emplea sólo, dada su construcción es un mal indicador de cambios o semejanzas estructurales. En esta línea, las respuestas obtenidas indicarán que, por ejemplo, si cambian las condiciones de transporte (oferta), asumiendo que existe petróleo que explotar (insumo), la producción aumentaría en un 1\% (para abastecer la demanda), luego para que ello ocurra deberá ser modificada la oferta del sector transporte (y no la rama petróleo), y las consecuencias serán

13 Al respecto sólo indicar que la revista Economics Systems Research lleva editado 20 números con 4 publicaciones por volumen, en donde cada una alberga al menos 10 artículos científicos por publicación, esto es 40 al año, además, existen las Conferencias Internacionales que se celebran cada año con más de 100 publicaciones científicas cada uno (este año corresponde la versión XVI a realizarse en Brasil), y por último las III Jornadas Españolas Input-Output -las que se realizan cada 2 años-, esto da una idea del uso, recurrencia y variedad de aplicaciones que permite la técnica en cuestión. 
un nuevo ciclo económico en donde el vertebrado y entramado pasarán a depender de las consecuencias del aumento en la explotación del citado combustible, esto es, de los cambios que se experimentan tanto en la oferta como demanda del sector (petróleo), y a su vez, de la oferta y demanda que realiza el sistema productivo a la rama, no entenderlo así es errado. Es decir, en el análisis realizado se incluye la oferta y demanda que se realiza del sector, y a su vez de la economía a la rama, por tanto, asumir que no se dice nada de la oferta o la demanda de la rama y del entorno sobre la rama, independientemente del stock disponible para su proceso, es ignorar el procedimiento utilizado.

Finalmente, y en alusión a la elección de las regiones tratadas, si bien es cierto son distintas, no sólo en términos estructurales sino que también dadas sus condiciones geográficas y lo insertada que las mismas pueden estar con respecto a otras regiones o, países, la ventaja del modelo aplicado y los aportes que a él se realizan, son para detectar precisamente entre otras estas diferencias, las cuales quedan manifestadas a partir del momento en que se utilizan tablas domesticas, luego argumentos tales cómo, la presencia de un corredor internacional de comercio, no obstaculiza el uso de la técnica escogida, sino que por el contrario la confirman, pues se trata precisamente de detectar estas diferencias, las que quedan reflejadas en las tablas input-output, independientemente de las diferencias que puedan existir en términos de transporte y tamaño de mercado, en este sentido, el propio Leontief argumentaba que, mientras más desarrollado es un país, más se parece su estructura interna -esto es, vertebrado y entramado-, a otros países desarrollados, esto ocurre precisamente por la determinación tecnológica que existe en los procesos industriales. En esta línea, si un sector y por ende una economía apunta a ello, más tendencia existirá por parte de las economías menos beneficiadas a imitar dichos procesos -independientemente de las diferencias en trasporte y tamaño de su mercado que puedan existir-, luego se valida el hecho de tener que comparar economías disímiles en pro de encontrar y crear similitudes y políticas que apunten a determinados logros económicos.

Desde otra perspectiva y, en alusión al punto precedente, se debe agregar que dado el hecho indudable de contar con nuevas y mejores estadísticas regionales, las cuales manifiestan una evidencia empírica clara de las diferencias de desarrollo socioeconómico entre las regiones, hace que estos estudios no sólo sean cada vez más recurrentes sino que también necesarios, pues ayudan a explicar las razones fundamentales de ello $y$, permiten diseñar estrategias y medidas dirigidas específicamente a estimular el crecimiento (regional), allí donde este es inferior o, bien, corregir los desequilibrios que puedan existir o, imitar determinadas políticas de desarrollo que auguran cierto grado de éxito (aunque no lo garanticen). En este sentido, el empleo de la técnica utilizada y los cambios propuestos ayudan a dar respuesta a ello, aún cuando existen otros modelos como los de Solow (1956) ${ }^{14}$, en donde se pone énfasis a los papeles que juegan los factores de oferta en el crecimiento, o bien, los modelos derivados de él como los de crecimiento endógeno, estos no son adecuados pues no explican en detalle cuáles son las causas reales de estas diferencias, como tampoco lo hacen otros modelos de corte keynesiano, que explican el crecimiento por la influencia de los factores de demanda, e.g. base exportadora o renta interregional, o la teoría de polos de crecimiento o la causación acumulativa, esto porque ninguno de ellos explica en profundidad los motivos de estas diferencias, aunque claro está, ellos explican en parte tales diferencias en términos de unas pocas variables, por mencionar algunas, capital, trabajo, salarios, renta y gastos regional, importaciones y exportaciones, etc, pero no entregan el detalle que permite el análisis input- output, por tanto, se cree que la técnica escogida es la más adecuada a los fines establecidos, pues explica con más profundidad las causas de las diferencias para estas regiones.

\section{AGRADECIMIENTOS}

El autor desea agradecer los comentarios y sugerencias vertidos por los árbitros anónimos y editores de esta revista. De igual modo, desea hacer presente su reconocimiento a Patricio Aroca (Universidad Católica del Norte) y a, Marcello Sasso (Universidad de Magallanes). Cualquier error u omisión es responsabilidad del infrascrito, no comprometiendo por tanto a los anteriores, o a esta revista, ni al Grupo ICM, o a la Universidad de Magallanes.

14 Solow, R. M. A contribution to the theory of economic growth. Quarterly Journal of Economics, 70: 65- 94, 1956. 


\section{BIBLIOGRAFÍA}

AROCA, Patricio. Impacts and Development in Local Economies Based on Mining: The case of the Chilean II region. Resources Policy, 27: 119- 134, 2001.

AROCHE, Fidel. Desintegración en la Estructura Productiva Mexicana y el Empleo. Los Coeficientes Importantes y su Importancia en la Integración. En: I Jornadas de Análisis Input-Output, Universidad de Oviedo, España, 22-23 de Septiembre, 2005.

- Structural Transformations and Important Coefficients in the North American Economies. Economic Systems Research, 14(3):257-273, 2002.

- Important Coefficients and Structural Change: A multi-layer approach. Economic Systems Research, 8(3):235-247, 1996.

Banco Central de Chile. Cuentas Nacionales: 2003- 2007. Banco Central de Chile, Santiago, 2008.

- Cuentas Nacionales: 2003- 2006. Banco Central de Chile; Santiago, 2007.

- Cuentas Nacionales: 1996- 2005. Banco Central de Chile; Santiago, 2006.

BULLARD, Clark and SEBALD Anthony. Effects of Parametric Uncertainty and Technological Change on Input-Output Models. The Review of Economic and Statistics, 59(1):75-81, 1977.

CHENERY, Hollis and WATANABE, Tsunehiko. An International Comparison of the Structure of Production. Econométrica, 26(4): 487-521, 1958.

De BOER, Paul. Additive Structural Decomposition Analysis and Index Number Theory: An Empirical Application of the Montgomery Decomposition. Economic Systems Research, 20 (1): 97- 109, 2008.

DIETZENBACHER, Erik and LINDEN, Jan van der. Sectoral and Spatial Linkages in the EC Production Structure. Journal of Regional Science, 37(2): 235-257, 1997.

EVANS, Duane. The Effect of Structural Matrix Errors on Interindustry Relations Estimates. Econometrica, 22(4):461-480, 1954.

Le MASNE, Pierre. Le système productif français face a ses voisins européens. Troisième Colloque de Comptabilité Nacionale, París, 12-14 diciembre de 1988.

LÓPEZ, Ana María, y PULIDO, Antonio. Análisis de las Interrelaciones Sectoriales en España. Economía Industrial, 290: 167-178, 1993.

GHOSH, Santadas and ROY, Joyashree. Qualitative Input-Output Analysis of the Indian Economic Structure. Economic Systems Research, 10(3):263-272, 1998.
HAIR, Joseph, ANDERSON Rolph, TATHAM Ronald y William BLACK. Análisis Multivariante. Quinta edición, Madrid, Ed. Prentice Hall, 2000.

HEWINGS, Geoffrey, FONSECA, Manuel and SONIS, Michael. Key Sectors and Structural Change in the Brazilian Economy: A comparison of alternative approaches and their policy implications. Journal of Policy Modeling, 11(1):67-90, 1989.

HIRSCHMAN, Albert. The Strategy of Economic Development. New Haven, Connecticut, USA, Yale University Press, 1958.

HOLUB, H-W und SCNNABL, Hermann. Input-OutputRechnung: Input-Output-Analyse. München, Wien: Oldenbourg, 1994.

JALILI, Ali Reza. Comparison of Two Methods of Identifying Input-Output Coefficients for Exogenous Estimation. Economic Systems Research, 12(1):113-129, 2000.

Le MASNE, Pierre. Le système productif français face a ses voisins européens. Troisième Colloque de Comptabilité Nacionale, París, 12-14 diciembre de 1988.

LÓPEZ, Ana María, y PULIDO, Antonio. Análisis de las Interrelaciones Sectoriales en España. Economía Industrial, 290: 167-178, 1993.

NAKAJIMA, Akiro. Total Labour Requirements and Value Added Productivity of Labour in the Process of Economic Development. Economic Systems Research, 20 (3): 319- 330, 2008.

MIDEPLAN. Aproximación a las Economías Regionales con base en las Matrices de Insumo Producto Regionales del Año 1996. Santiago, Chile, Ministerio de Planificación, 2005, pp. 211

OOSTERHAVER, Jan; Dirk STELDER and Satoshi INOMATA. Estimating International Interindustry Linkages: Non-survey Simulations of the Asian-Pacific Economy. Economic Systems Research, 20 (4): 395- 414, 2008.

PULIDO, Antonio; y FONTELA, Emilio. Análisis Input-Output. Modelos Datos y Aplicaciones. España. Editorial Pirámide, 1993.

RASMUSSEN, Paul Noregaard. Studies in Inter-Sectoral Relations. Amterdam, North- Holland P. C. 1956.

ROBLES, Luís y SANJUÁN, Jesús. Sectores y Clusters Claves en la Economía Española. ICE Revista de Economía, Ministerio de Industria, Turísmo y Comercio, Gobierno de España, 843: 183-207, 2008.

- Coefficient Stability and Structural Change in the Spanish Economy. Econ Change, 40: 387- 409, 2007.

- Análisis Comparativo de la Tablas Input- Output en el Tiempo. Estadística Española, 47(158): 143- 177, $2005 a$. 
- Key Sectors: Big Coefficients and Important Coefficients in Spain. In: I Jornadas de Análisis Input-Output, Universidad de Oviedo, España, 22-23 de Septiembre, 2005b.

SCHINTKE, Joachim. Fehlersimulationen Mit Input-Ouput-Tabellen des Statitischen Bundesamtes. Vierteljahrshefte zur Wirtschaftsforschung, 3:314-330, 1984.

SCHINTKE, Joachim and STÄGLIN Reiner. Important Input Coefficients in Market Transaction Tables and Production Flow Tables. In: CIASCHINI, M. (Ed), Input-Output Analysis, Chapman and Hall, New York, pp. 43-60, 1988.

SCHNABL, Hermann. The Evolution of Production Structures, Analyzed by a Multi-Layer Procedure. Economic Systems Research, 6(19):51-69, 1994.

SIEBE, Thomas. Important Intermediate Transactions and Multi-sectoral Modelling. Economic Systems Research, 8(2):183-194, 1996.

SEBALD, Anthony. An Analysis of the Sensitivity of Large Scale Input-Output Models to Parametric Uncertainties. Center for Advanced Computation, document no. 122, University of Illinois al Urbana, 1974.

SHERMAN, Jack and MORRISON, Winifred. Adjustment of and Inverse Matrix Correspondinding to a Change in One Element of a Given Matrix. The Annals of Mathematical Statistics, 21(1):124-127, 1950.

SONIS, Michael and HEWINGS, Geoffrey. Coefficient Change in Input- Output Models: Thery and applications. Economic Systems Research. 4: 143-157, 1992.

- Fields of Influence and Extended Input- Output Analysis: A theorical account. In: J.H.Ll. Dewhurst, R.C. Jensen and G.J.D. Hewings (eds) Regional Input- Output Modeling - New Developments and Interpretations (Aldershot, Avebury), pp. 141-158, 1991.

- Error and sensitivity Input- Output Analysis: A new approach. In: R.E. Miller, K.R. Polenske and A.Z. Rose (eds) Frontiers of Input- Output Analysis (New York, Oxford University Press), pp. 232-244, 1989.

SONIS, M., J. GUILHOTO, G. HEWINGS, and E. MARTINS. Key Sectors, and Structural Change: Some New Perspectives. The Developing Economics, 33(3): 233-270, 1995.

SOZA, Sergio. Análisis Estructural Input- Output: Una comparativa para el caso chileno. En: XI Reunión de Economía Mundial, Universidad de Huelva, España, 20- 22 de Mayo, 2009a.

- Sectores Calves y Campos de Influencia en Magallanes. En: XI Reunión de Economía Mundial, Universidad de Huelva, España, 20- 22 de Mayo, 2009b.

- Análisis Comparativo para la Economía Magallánica desde la Perspectiva del Enfoque Input-Output. Revista de Análisis Económico, 23(2):79-102, 2008a

- Interrelaciones Sectoriales en Magallanes: En busca de su estructura económica. Magallania, 36(1):79-102, 2008 b.

- Análisis Estructural Input-Output: Antiguos problemas y nuevas soluciones. Tesis (Doctorado en Economía Aplicada). Oviedo, España, Universidad de Oviedo, Departamento de Economía Aplicada, 280 p., 2007.

SOZA, Sergio y Carmen RAMOS. Replanteamiento del análisis estructural a partir del análisis factorial. Una aplicación a economías europeas. Estudios de Economía Aplicada, 23(2): 363-384, 2005.

TARANCÓN, Miguel Ángel. Técnicas de Análisis Económico Input-Output. Alicante, Editorial Club Universitario, 2003, 269p.

WAUGH, Frederick. Inversion of the Leontief Matriz by Power Series. Econometrica, 18(2):142-154, 1950.

WEST, Guy. Sensitivity and Key sector Analysis in Input-output Models. Australian Economic Papers, 21:365-378, 1982. 
Anexo 1A: Relación entre el número de sector y lo que representa.

\begin{tabular}{|c|c|}
\hline Rama/ Sector Número & Rama \\
\hline 1 & Productos Agrícolas \\
\hline 2 & Frutas \\
\hline 3 & Ganado; productos pecuarios y carne \\
\hline 4 & Productos silvícolas; Maderas y Papel \\
\hline 5 & Pescados y mariscos frescos y Productos del Mar Industrializados \\
\hline 6 & Petróleo crudo y combustibles y lubricantes \\
\hline 7 & Cobre \\
\hline 8 & Otros minerales \\
\hline 9 & Conservas de frutas y vegetales \\
\hline 10 & Aceites y grasas \\
\hline 11 & Productos lácteos \\
\hline 12 & Productos de molinería; Pan, fideos y pastas \\
\hline 13 & Otros productos alimenticios \\
\hline 14 & Alimentos para animales \\
\hline 15 & Licores; cervezas; bebidas analcohólicas y vinos \\
\hline 16 & Productos del tabaco \\
\hline 17 & Productos textiles, prendas de vestir, cuero y calzado \\
\hline 18 & Impresos y grabaciones \\
\hline 19 & Productos químicos, de caucho y de plástico \\
\hline 20 & Vidrio, productos de vidrio, y productos de minerales no metálicos \\
\hline 21 & Productos básicos de hierro y acero y productos metálicos \\
\hline 22 & Maquinaria y equipo eléctrico y no eléctrico \\
\hline 23 & Muebles \\
\hline 24 & Otros productos manufactureros \\
\hline 25 & Electricidad \\
\hline 26 & Gas y Agua \\
\hline 27 & Productos de la Construcción \\
\hline 28 & Servicios comerciales \\
\hline 29 & Servicios de hotelería y de restaurantes \\
\hline 30 & Servicios de transporte ferroviario y caminero \\
\hline 31 & Servicios de transporte marítimo y aéreo \\
\hline 32 & Servicios conexos de transporte \\
\hline 33 & Servicios de comunicaciones \\
\hline 34 & Servicios financieros y de seguros \\
\hline 35 & Servicios empresariales \\
\hline 36 & Servicios inmobiliarios y de propiedad de vivienda \\
\hline 37 & Servicios de administración pública \\
\hline 38 & Servicio de educación pública y privada \\
\hline 39 & Servicio de salud pública y privada \\
\hline 40 & Servicios de esparcimiento y otros servicios \\
\hline
\end{tabular}

Fuente: MIDEPLAN (2005). 
Anexo 2A: Número de actividades más importantes según relación de compra y venta entre ellas $\left(\mathbf{r}_{\mathrm{ij}} \leq 5\right)$.

\begin{tabular}{|c|c|c|c|c|c|c|c|c|c|c|c|}
\hline \multirow[b]{2}{*}{ Rama } & \multicolumn{2}{|c|}{ Tarapacá } & \multicolumn{2}{|c|}{ L. B. O'Higgins } & \multicolumn{2}{|c|}{ Bío- Bío } & \multicolumn{2}{|c|}{ Los Lagos } & \multicolumn{2}{|c|}{ Magallanes } & \multirow[b]{2}{*}{ Rama } \\
\hline & Compra & Venta & Compra & Venta & Compra & Venta & Compra & Venta & Compra & Venta & \\
\hline 1 & & & & 1 & & 1 & & 1 & & 1 & 1 \\
\hline 2 & & & & & & 1 & & & & & 2 \\
\hline 3 & 1 & 1 & 3 & 1 & 2 & 2 & 3 & 2 & 2 & 1 & 3 \\
\hline 4 & & 1 & 1 & 2 & 2 & 1 & 1 & 1 & 1 & 1 & 4 \\
\hline 5 & 1 & 1 & & 1 & 2 & 1 & 2 & 1 & 1 & 1 & 5 \\
\hline 6 & 1 & 1 & 2 & 3 & & & 1 & 1 & 5 & 1 & 6 \\
\hline 7 & 1 & & 1 & & 1 & 1 & 1 & 1 & 1 & 1 & 7 \\
\hline 8 & & & & & & 1 & & & & & 8 \\
\hline 9 & & & & & 1 & & & & & & 9 \\
\hline 10 & & & 1 & 1 & & & & 1 & & 1 & 10 \\
\hline 11 & & & & & 1 & & 1 & & 1 & 1 & 11 \\
\hline 12 & & & & & & & & & & 1 & 12 \\
\hline 13 & & & & & 1 & & & 1 & & 1 & 13 \\
\hline 14 & 1 & 1 & 2 & 1 & & 1 & 3 & 2 & 1 & 1 & 14 \\
\hline 15 & & & & & & & & & & & 15 \\
\hline 16 & 1 & 1 & & & 1 & 1 & 1 & 1 & 1 & 1 & 16 \\
\hline 17 & & & & & & & & & & & 17 \\
\hline 18 & & 1 & & 1 & & 1 & & & & 2 & 18 \\
\hline 19 & & & 1 & & & & & & & & 19 \\
\hline 20 & & 1 & & 1 & & 1 & & 1 & & 1 & 20 \\
\hline 21 & & & & 1 & & & & 1 & & 1 & 21 \\
\hline 22 & & & & & & & & & & & 22 \\
\hline 23 & & & & & & & & & & & 23 \\
\hline 24 & & & & & & 1 & & 1 & & & 24 \\
\hline 25 & & 2 & & 1 & 1 & & & & & & 25 \\
\hline 26 & & & & & & & & & & & 26 \\
\hline 27 & 3 & & 2 & & 1 & & 2 & & 2 & & 27 \\
\hline 28 & 3 & & & & 1 & & & & 1 & & 28 \\
\hline 29 & & & 1 & & & & & & 2 & & 29 \\
\hline 30 & & 1 & & & & 2 & & & & 1 & 30 \\
\hline 31 & 1 & 1 & 1 & 1 & 1 & 1 & 1 & 1 & & 1 & 31 \\
\hline 32 & & & & 1 & & & & & & & 32 \\
\hline 33 & & & & & & & & & & & 33 \\
\hline 34 & & & & & & & & & & & 34 \\
\hline 35 & 1 & 2 & 1 & & 1 & & & & 1 & 1 & 35 \\
\hline 36 & & & & & & & & & & & 36 \\
\hline 37 & & & & & & & & & & & 37 \\
\hline 38 & & & & & & & & & & & 38 \\
\hline 39 & & & & & & & & & & & 39 \\
\hline 40 & & & & & & & & & & & 40 \\
\hline
\end{tabular}

Fuente: Propia. 
Anexo 2B: Campos de Influencia para las Distintas Regiones ${ }^{15}$ :

\begin{tabular}{|c|c|}
\hline De Tarapacá & Del Libertador Bernardo O' Higgins \\
\hline 용 & 을 \\
\hline Del Bío- Bío & De Los Lagos \\
\hline$\frac{2}{2}$ & $\begin{array}{l}6 \\
8 \\
8 \\
8 \\
8 \\
8\end{array}$ \\
\hline \multicolumn{2}{|l|}{ De Magallanes } \\
\hline$\frac{3}{3}$ & \\
\hline
\end{tabular}

Fuente: Propia

15 Con la finalidad de facilitar la lectura, el primer sector se presenta con otro color, de igual forma, se indica que, el orden de las ramas, sigue el sentido de un reloj tradicional. 
Anexo 3A: Matriz de distancias

\begin{tabular}{|l|r|r|r|r|r|}
\hline Caso & \multicolumn{5}{|c|}{ Archivo matricial de entrada } \\
\hline & Puntua(R1) & Puntua(R6) & Puntua(R8) & \multicolumn{1}{c|}{ Puntua(R10) } & Puntua(R12) \\
\hline Puntua(R1) &, 000 & 61,513 & 70,639 & 64,672 & 46,863 \\
Puntua(R6) & 61,513 &, 000 & 47,897 & 42,712 & 47,013 \\
Puntua(R8) & 70,639 & 47,897 &, 000 & 26,884 &, 000 \\
Puntua(R10) & 64,672 & 42,712 & 26,884 & 57,846 \\
Puntua(R12) & 46,863 & 47,013 & 67,846 & 55,915 &, 000 \\
\hline
\end{tabular}

Fuente: Propia

Anexo 3B: Dendrograma

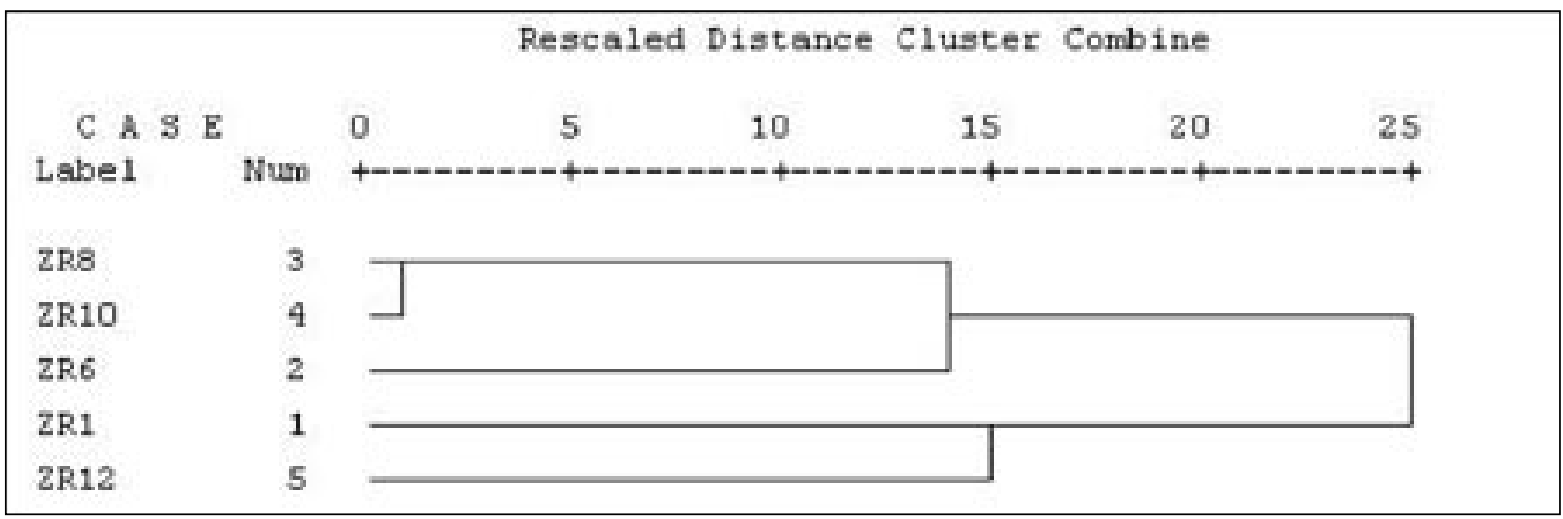

Fuente: Propia

Anexo 3C: Diagrama de témpanos vertical

\begin{tabular}{|c|c|c|c|c|c|c|c|c|c|}
\hline $\begin{array}{c}\text { Número de } \\
\text { conglomerados }\end{array}$ & \multicolumn{9}{|c|}{ Caso } \\
\hline & Puntua(R10) & & Puntua(R8) & & Puntua(R6) & & Puntua(R12) & Puntua(R1) \\
\hline 1 & $\mathrm{X}$ & $\mathrm{X}$ & $\mathrm{X}$ & $\mathrm{X}$ & $\mathrm{X}$ & $\mathrm{X}$ & $\mathrm{X}$ & $\mathrm{X}$ & $\mathrm{X}$ \\
2 & $\mathrm{X}$ & $\mathrm{X}$ & $\mathrm{X}$ & $\mathrm{X}$ & $\mathrm{X}$ & & $\mathrm{X}$ & $\mathrm{X}$ & $\mathrm{X}$ \\
3 & $\mathrm{X}$ & $\mathrm{X}$ & $\mathrm{X}$ & $\mathrm{X}$ & $\mathrm{X}$ & & $\mathrm{X}$ & & $\mathrm{X}$ \\
4 & $\mathrm{X}$ & $\mathrm{X}$ & $\mathrm{X}$ & & $\mathrm{X}$ & & $\mathrm{X}$ & & $\mathrm{X}$ \\
\hline
\end{tabular}

Fuente: Propia 
